Ann. Génét. Sél. anim., I972, 4 (3), 355-36I.

\title{
MODE DE TRANSMISSION D'UNE FUSION CENTRIQUE DANS LA DESCENDANCE D'UN BOUC (CAPRA HIRCUS L.) HÉTÉROZYGOTE
}

\author{
C. P. POPESCU \\ avec la collaboration technique de Jeannine Boscher \\ U. N.C.E.I.A. et Laboratoire de Génétique factorielle, \\ Centre national de Recherches zootechniques, I. N.R. A., \\ 78 - Jouy-en-Josas \\ RÉSUMÉ
}

L'étude porte sur 19 mâles et I I femelles descendant d'un bouc (Capra hircus L.) hétérozygote pour une fusion centrique: I 4 mâles et 6 femelles ont été trouvés porteurs de l'anomalie. La fusion centrique s'est produite entre deux acrocentriques avec la formation d'un nouveau chromosome submétacentrique et la réduction du nombre de base à 59. Aucun animal homozygote pour cette fusion n'a été trouvé et tous les animaux présentaient des caryotypes équilibrés. Les mesures ont été pratiquées en vue d'établir la position des chromosomes fusionnés dans l'idiogramme.

\section{IN'TRODUCTION}

Le caryotype normal de la chèvre (Capra hircus L.) est constitué de 6o chromosomes. Tous les autosomes ainsi que le chromosome $\mathrm{X}$ sont acrocentriques et le chromosome $\mathrm{Y}$, lui, est métacentrique et le plus petit du complément. Le chromosome $\mathrm{X}$ serait, selon EvaNs (I965) un des trois premiers grands acrocentriques. Les études cytogénétiques effectuées chez cette espèce concernent surtout le phénomène d'intersexualité lié à la présence du gène motte $P$, ou au free-martinisme (BASRur et Coubrough, I964; BASRur et KanaGawa, I968; CORTEEI, et al., I969) et sur une translocation de type robertsonien présente dans la race Saanen. Le premier cas de fusion centrique décrit chez la chèvre était représenté par un animal porteur d'une mosaïque cellulaire $\mathrm{XX} / \mathrm{XY}$ (PADEH et al., I965) apparue vraisemblablement par une double fécondation et dont la population XX contenait deux chromosomes fusionnés. La même anomalie est ensuite étudiée dans la descendance d'une femelle hétérozygote (Sol,LER et al., I966) ; récemment, les mêmes auteurs, dans une série de croisements expérimentaux, analysent son mode de transmission (PADEH et al., I97I). 
En France, une fusion centrique de même type a été trouvée chez un mâle de race Saanen (HuLOT, I969). Dans cet article nous présentons les résultats d'une étude sur son mode de transmission dans la descendance de 1970 de cet animal. Dans une publication ultérieure seront présentés les résultats concernant les effets de cette anomalie sur la fertilité.

\section{MATÉRIEL ET MÉTHODES}

L'animal trouvé porteur d'une fusion centrique a eu en I970, I9 descendants mâles et I7 descendants femelles issus d'accouplements avec des femelles phénotypiquement normales et supposées normales du point de vue chromosomique. Nous avons pu étudier les chromosomes de tous les descendants mâles et de i I descendants femelles sur les I 7 .

Des cultures de sang total ont été pratiquées selon la méthode de de Grouchy et al. (1964), modifiée (PoPESCU, 1968). Environ 25 métaphases ont été étudiées pour chaque animal et les meilleures ont été photographiées au grossissement de I $250 \times$ sur une pellicule Kodak-Microfilm. Un idiogramme a été établi sur 5 cellules portant la fusion centrique, mesurées selon une méthode décrite antérieurement (Popescu, I969). En raison de la difficulté d'identification du chromosome $\mathrm{X}$ les valeurs relatives ont été calculées, non pas pour le complément haploïde mais pour tous les chromosomes du complément diploïde. Elles sont exprimées comme part pour mille de la longueur totale du complément diploïde. La moyenne, la variance et l'erreur standard de la moyenne ont été calculées pour chaque chromosome.

Les chromosomes méiotiques ont été étudiés sur trois mâles dont I normal et deux porteurs de la fusion centrique. Les préparations ont été réalisées à partir de fragments de testicule, selon la technique d'Evans et al., (1964).

\section{RÉSULTATS ET DISCUSSION}

Sur les I9 descendants mâles, I4 ont été trouvés porteurs de la fusion centrique (tabl. I) tandis que parmi les femelles seulement 6 sur II la portent (fig. I).

TABLEAU I

La distribution de la fusion centrique dans la descendance d'un mâle hétérozygote

\begin{tabular}{|c|c|c|c|}
\hline \multirow{2}{*}{ Distribution } & \multicolumn{2}{|c|}{ Caryotype } & \multirow{2}{*}{ Tota } \\
\hline & $2 n=60$ & $2 n=58+\mathrm{T}$ & \\
\hline \multicolumn{4}{|l|}{ Descendance $\widehat{\sigma}$} \\
\hline Observée........ & 5 & 14 & \\
\hline Escomptée .......... & 9,5 & 9,5 & 19 \\
\hline \multicolumn{4}{|l|}{ Descendance } \\
\hline Observée............. & 5 & 6 & \\
\hline Escomptée .......... & 5,5 & 5,5 & 11 \\
\hline \multicolumn{4}{|c|}{ 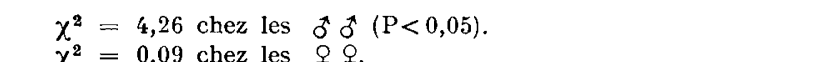 } \\
\hline
\end{tabular}


MODE DE TRANSMISSION D'UNE FUSION CENTRIQUE

357

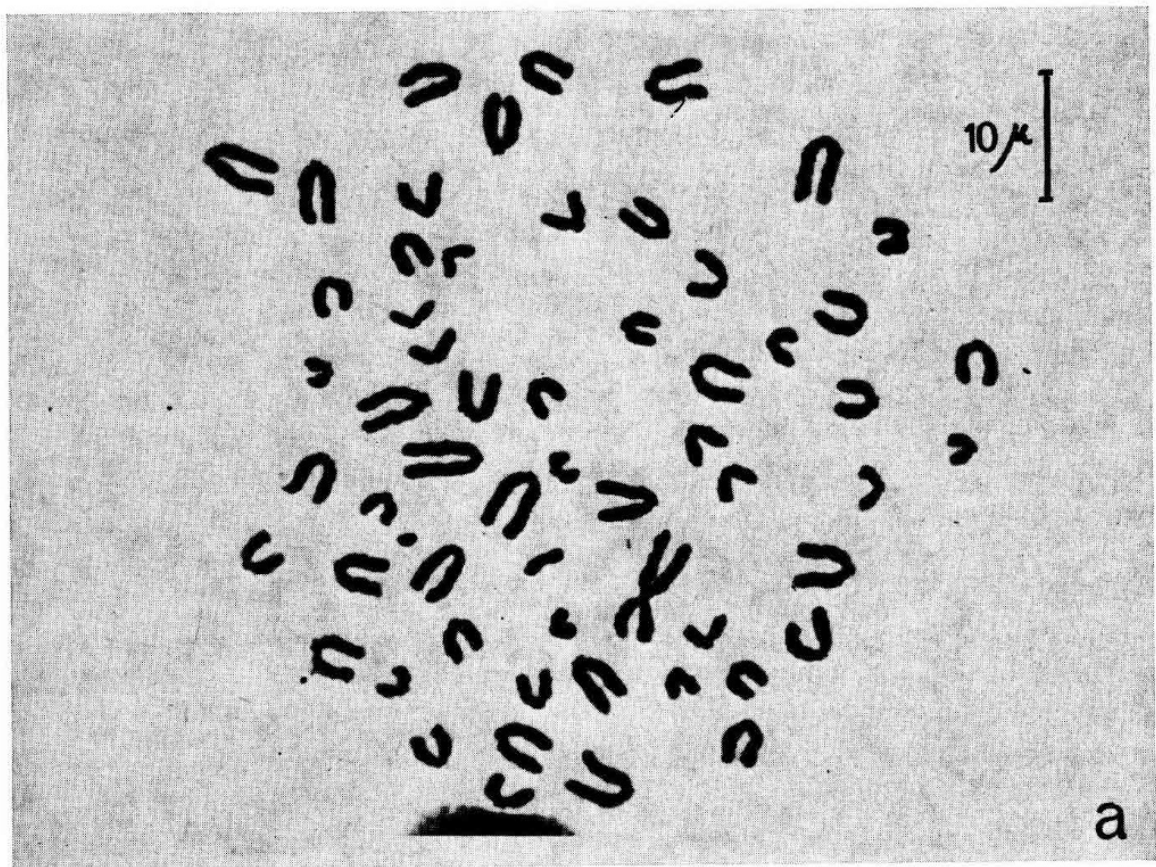

ha $\cos$ an $\operatorname{an}$ an on $\cos$ an $\mathrm{n} n \mathrm{n}$ bn $n \cap$ n no $n n$ na on an on an no na an an a a na a n a a $n$. b

a) Métaphase mâle portant la fusion centrique
b) Le caryotype de la même cellule 
Tous les animaux étudiés, normaux ou hétérozygotes pour la fusion centrique, présentaient des caryotypes équilibrés. On remarque 1'absence totale d'individus homozygotes pour cette anomalie. L'idiogramme présenté dans le tableau 2 a été établi en vue de déterminer les chromosomes impliqués dans la fusion centrique. Selon les valeurs obtenues, celle-ci se serait produite entre un acrocentrique de la $2^{\mathrm{e}}$ paire et un autre, plus petit, appartenant à la $\mathrm{I} 3^{\mathrm{e}}$ paire.

TABLEAU 2

Les moyennes des longueurs relatives de chromosomes diploïdes exprimées comme part pour mille

\begin{tabular}{|c|c|c|c|c|c|}
\hline No & $\begin{array}{l}\text { Longueur rel. } \\
\text { moyenne }\end{array}$ & Erreur standard & No & $\begin{array}{l}\text { Longueur rel. } \\
\text { moyenne }\end{array}$ & Erreur standard \\
\hline 1 & 29,67 & 0,58 & 31 & 15,62 & 0,27 \\
\hline 2 & 29,11 & 0,54 & 32 & 15,14 & 0,12 \\
\hline 3 & 27,80 & 0,40 & 33 & 14,69 & 0,20 \\
\hline 4 & 27,23 & 0,67 & 34 & 14,54 & 0,17 \\
\hline 5 & 26,22 & 0,64 & 35 & 14,40 & 0,22 \\
\hline 6 & 24,96 & 0,65 & 36 & 14,08 & 0,15 \\
\hline 7 & 24,44 & 0,74 & 37 & 13,66 & 0,15 \\
\hline 8 & 23,75 & 0,49 & 38 & 13,50 & 0,21 \\
\hline 9 & 23,34 & 0,26 & 39 & 13,31 & 0,24 \\
\hline 10 & 23,12 & 0,28 & 40 & 13,14 & 0,24 \\
\hline 11 & 22,57 & 0,23 & 41 & 12,85 & 0,35 \\
\hline 12 & 22,24 & 0,34 & 42 & 12,41 & 0,28 \\
\hline 13 & 21,88 & 0,29 & 43 & 11,95 & 0,33 \\
\hline 14 & 21,73 & 0,29 & 44 & 11,80 & 0,33 \\
\hline 15 & 21,56 & 0,24 & 45 & 11,50 & 0,24 \\
\hline 16 & 21,17 & 0,20 & 46 & 11,38 & 0,17 \\
\hline 17 & 20,77 & 0,15 & 47 & 10,85 & 0,34 \\
\hline 18 & 19,94 & 0,17 & 48 & 10,41 & 0,26 \\
\hline 19 & 19,60 & 0,22 & 49 & 10,30 & 0,24 \\
\hline 20 & 19,23 & 0,16 & 50 & 10,20 & 0,25 \\
\hline 21 & 18,81 & 0,19 & 51 & 9,97 & 0,24 \\
\hline 22 & 18,53 & 0,31 & 52 & 9,88 & 0,21 \\
\hline 23 & 18,42 & 0,30 & 53 & 9,61 & 0,31 \\
\hline 24 & 18,09 & 0,29 & 54 & 9,32 & 0,25 \\
\hline 25 & 17,37 & 0,22 & 55 & 9,03 & 0,31 \\
\hline 26 & 17,27 & 0,19 & 56 & 8,78 & 0,30 \\
\hline 27 & 16,81 & 0,19 & 57 & 8,30 & 0,36 \\
\hline 27 & 16,81 & 0,19 & 57 & 8,30 & 0,36 \\
\hline 28 & 16,47 & 0,24 & & & \\
\hline 29 & 16,22 & 0,29 & Chr. fusionné & Bras long & \\
\hline \multirow[t]{2}{*}{30} & 15,75 & 0,30 & & 27,28 & 0,69 \\
\hline & & & & $\begin{array}{c}\text { Bras court } \\
17,28\end{array}$ & 0,92 \\
\hline
\end{tabular}

Dans les préparations méiotiques provenant des deux animaux hétérozygotes pour la fusion centrique (fig. 2) nous avons toujours identifié, à la métaphase I, un trivalent qui contenait le chromosome fusionné et, d'autre part, le bivalent sexuel, facilement reconnaissable à cause de son état hétérochromatique. 
Selon l'idiogramme le grand acrocentrique impliqué dans la fusion a une taille voisine du chromosome $\mathrm{X}$. Mais la présence du bivalent sexuel et du trivalent dans les métaphases I indique, sans aucun doute, que la fusion centrique concerne deux autosomes et exclut la participation du chromosome $\mathrm{X}$.

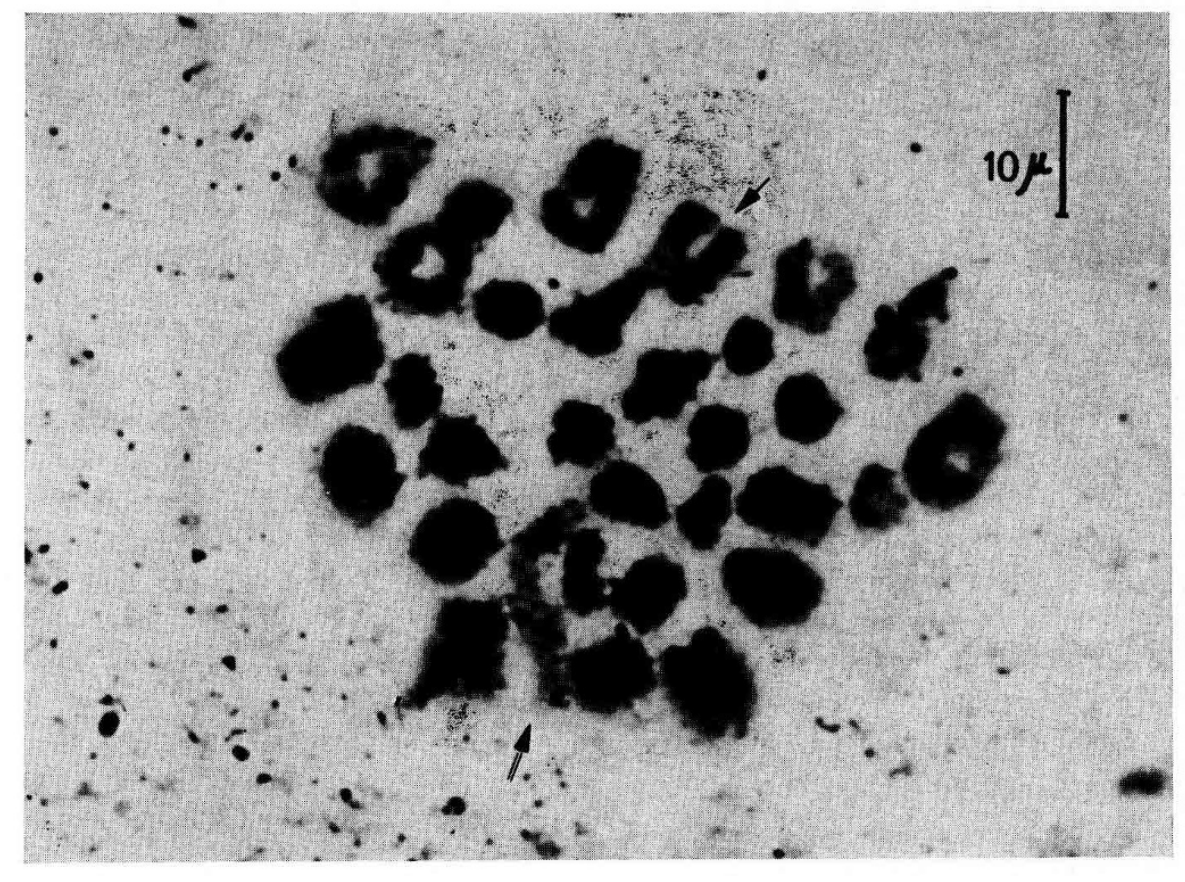

Fig. 2. - Diacinèse

Le trivalent est indiqué par une flèche simple et le bivalent sexuel par une flèche double

D'après les valeurs relatives contenues dans l'idiogramme, il semble, comme nous l'avons indiqué plus haut, que les deux chromosomes fusionnés appartiennent à $1 \mathrm{a} 2^{\mathrm{e}}$ et respectivement, $1 \mathrm{a} \mathrm{I}^{\mathrm{e}}$ paire autosomale, mais leur position exacte dans la série décroissante est difficile à préciser en raison des erreurs de mesure et du caractère particulier des chromosomes de cette espèce. En effet, les chromosomes de la chèvre sont extrêmement difficiles à étudier à cause de leur morphologie identique et l'idiogramme, ainsi que chez les bovins, est basé uniquement sur les longueurs relatives. Le deuxième élément de reconnaissance, la position du centromère, qui intervient normalement dans un idiogramme est dans ce cas sans valeur, puisque tous les autosomes sont acrocentriques. Dans les publications précédentes (PADEH et al., I965; SOLLER et al., I966; PADEH et al., I97I), il n'existe pas d'indication sur les chromosomes impliqués dans la fusion, mais il est possible qu'elle concerne les mêmes chromosomes. Chez les bovins, une fusion du même genre, produite entre le plus grand et le plus petit autosome, présente dans plusieurs races et dans des pays différents, pourrait avoir une origine commune (PoPESCU, I97I).

Dans la transmission de cette anomalie on remarque une prédominance nette d'hétérozygotes par rapport aux normaux dans la descendance mâle, tandis que chez 
les femelles ce rapport est à peu près égal (I : I). La même observation a été faite par HaMERTON (I965) chez l'homme dans le cas d'une fusion D-G. L'auteur suppose que les gamètes mâles porteurs de l'anomalie auraient un certain avantage adaptatif, par rapport aux normaux. Cette explication pourrait être aussi variable pour les chèvres et pour d'autres espèces. On connaît des espèces pour lesquelles un polymorphisme chromosomique dû à une translocation représente une voie de spéciation (MatThey, I970).

Parmi les descendants de ce mâle hétérozygote pour la fusion centrique, nous avons remarqué également l'absence totale de caryotypes non équilibrés. Cette même constatation a été faite antérieurement chez les bovins (Gustavsson, 1969) et chez les chèvres (PADEH et al., I97I). HAMERTON (I965) chez les humains, bien qu'il trouve un certain pourcentage de caryotypes non équilibrés constate, lui aussi, un déficit de ces derniers et une prédominance des caryotypes équilibrés. Pourtant, si la ségrégation se fait au hasard, les chances théoriques de l'apparition de tels gamètes sont les mêmes que pour les gamètes équilibrés porteurs de la translocation et normaux. L'orientation des homologues dans le trivalent, durant la $I^{r e}$ prophase méiotique, peut se faire d'une façon alternative, suivie par une ségrégation normale, ou adjacente, suivie d'une ségrégation anormale qui entraîne l'apparition de plusieurs types de gamètes non équilibrés. Il est admis qu'une certaine partie de ces gamètes donnerait, après la fécondation, des zygotes létaux qui augmenteraient l'incidence de la mortalité embryonnaire.

L'absence totale des caryotypes non équilibrés dans la descendance étudiée peut constituer un argument en faveur de l'existence, au niveau de la méiose ,d'un mécanisme qui favoriserait l'orientation alternative et la formation préférentielle des gamètes équilibrés.

Reçu pour publication en février 197\%.

\section{SUMMARY}

\section{THE TRANSMISSION OF A CENTRIC FUSION IN THE PROGENY} OF A HETEROZYGOTE HE-GOAT (CAPRA HIRCUS L.)

Cytogenetics studies were undertaken on the progeny of a Robertsonian type translocation carrier male. Out of $I_{9}$ males and 22 females offsprings examined, I 4 males and 6 females were found to be carrier of this type of translocation. It appears that two acrocentric chromosomes fuse to form a new submetacentric chromosome and thereby reduce the basic number of chromosomes to 59. No homozygote for this type of translocation was observed. All of the animals examined had balanced karyotypes. The abnormal chromosome was relocated in the meiotic preparations. Mesurements were made to the abnormal caryotypes with a view to establish an idiogram and define the particular chromosomes involved in the fusion.

\section{RÉFÉRENCES BIBLIOGRAPHIQUES}

Basrur P. K., Coubrough R. I., I964. Anatomical and cytological sex of a Saanen goat. Cytogenetics, 3, 4I4-426.

Basrur P. K., Kanagawa H., I968. Cytogenetic studies on intersex goats (abstr.). Canad J. Genet. Cytol., 10, 764 . 
Corteel J. M., Hulot Françoise, Courot M., Attal J., Philippon A., r969. Examens morphologiques, caryologiques, physiologiques et pathologiques de boucs stériles sans cornes. Ann. Génét. Sél. anim., 1, 341-348.

Evans H. J., I965. A simple microtechnique for obtaining human chromosome preparations with some comments on DNA replication in sex chromosomes of the Goat, Cow and Pig. Expt. Cell. Res., 38, 5 II-5I6.

Evans E. P., Brecton G., Ford C. E., I964. An air drying method for meiotic preparations from mammalian testis. Cytogenetics, 3, 289-294.

Grouchy J., De Roubin M., Passage E., I964. Microtechnique pour l'étude des chromosomes humains à partir d'une culture de leucocytes sanguins. Ann. Génét., \%, 45-46.

Gustavsson I., I969. Cytogenetics distribution and phenotypic effects of a translocation in swedish cattle. Hereditas, 63, 68-x69.

HaMerton J. L., I968. Robertsonian translocation in man : evidence for prezygotic selection. Cytogenetics, 7, 260-276.

Hulot Françoise, r969. Nouveau cas de fusion centrique chez la chèvre domestique (Capra hircus L.). Ann. Génét. Sél. anim., 1, I75-I76.

MAtthey R., I97o. L' "éventail robertsonien " chez les Mus/Leggada africains du groupe minutoides. musculoides. Rev. suisse Zool., 77, 625-629.

Padeh B., Wysoki M., Ayalon N., Soller M., 1965. An XX/XY hermaphrodite in the goat. Israel J. med. Sci., 5, I oO8-I oI2.

PADEH B., WySOKI M., SolleR M., I97I. Further studies on a Robertsonian translocation in the Saanen dairy goat. Cytogenetics, 10, 6I-69.

Popescu C. P., x 969 . Technica de microcultura din sange periferic pentru studiul cromosomilor la animale domestice. St. Cerc. Biol. Seria Zoologie, 20, 42I-422.

Popescu C. P., r969. Idiograms of Yak (Bos grunniens), cattle (Bos taurus) and their hybrid. Ann. Génét. Sél. anim., 1, 207-217.

Popescu C. P., I97I. Deux cas nouveaux de fusion centrique chez les bovins. Ann. Génét. Sél. anim., $4(52 \mathrm{I}-525)$

SOLleR M., WysoKi M., PADEH B., rg66. A chromosomal abnormality in phenotypically normal Saanen goats. Cytogenetics, 5, 88-93. 